\title{
Frequent respiratory events in postoperative patients aged 60 years and above
}

This article was published in the following Dove Press journal:

Therapeutics and Clinical Risk Management

26 August 2017

Number of times this article has been viewed

\author{
Suzanne JL Broens' \\ Xuan $\mathrm{He}^{1}$ \\ Rachel Evley ${ }^{2}$ \\ Erik Olofsen' \\ Marieke Niesters' \\ Ravi P Mahajan ${ }^{2}$ \\ Albert Dahan' \\ Monique van Velzen' \\ 'Department of Anesthesiology, \\ Leiden University Medical \\ Centre, Leiden, the Netherlands; \\ ${ }^{2}$ Nottingham University Hospital \\ NHS Trust, Queen's Medical Centre, \\ Nottingham, UK
}

\begin{abstract}
There is limited information on the occurrence of respiratory events in postoperative patients after discharge from the postanesthesia care unit. We studied the respiratory rate (RR) of 68 patients aged 60 years and above during the first 6 hours following elective surgery under general anesthesia to assess the frequency of respiratory events in the care unit and on the ward. RR was derived from the continuous RR counter RespiR8, measuring RR by quantifying the humidity of exhaled air. One-minute-averaged RRs were collected and analyzed to assess the frequency of postoperative bradypnea (RR 1-6 breaths/minute) and apnea (cessation of inspiratory flow $\geq 60$ seconds). Values were median (interquartile range) or mean (SD). The median RR was 13 (10-15) breaths/minute. In the 6-hour postoperative period, $78 \%$ and $57 \%$ of patients experienced at least one bradypnea or apnea event, respectively. A median of ten (3.5-24) bradypnea and three (1-11) apnea events were detected per patient. The occurrence of respiratory events in the postanesthesia care unit (PACU) was a predictor of events on the ward (bradypnea, $r^{2}=0.4, P<0.001$; apnea, $\left.r^{2}=0.2, P<0.001\right)$. Morphine consumption correlated weakly with respiratory events in the PACU, but not on the ward. Patients with apnea had significantly larger neck circumference than patients without (39.6 [0.7] versus 37.4 [0.8] cm, $P<0.05$ ). Bradypneic or apneic respiratory events are frequent in postoperative elderly patients and even occur relatively late after surgery. Continuous respiratory monitoring on the ward, especially in patients with risk factors, such as early occurrence of events, opioid use, and larger neck circumference, is likely warranted.
\end{abstract}

Keywords: aged, monitoring, physiologic, postoperative period, respiratory insufficiency, respiratory rate

\section{Introduction}

In current clinical practice, moderate-severe pain in postoperative patients is treated with potent opioids. While effective against pain in most patients, opioids come with serious side effects, of which opioid-induced respiratory depression is potentially life-threatening. ${ }^{1}$ In a review of the literature, we recently retrieved 132 case studies since 1980 in adult and pediatric patients that described the occurrence of postoperative respiratory depression from opioid treatment. ${ }^{2,3}$ Apart from these case studies, there was limited information on the prevalence of respiratory events in the postoperative period, with a few studies using continuous monitoring beyond measurements in the postanesthesia care unit (PACU) that showed frequent episodes of hypoxemia and/or bradypnea. ${ }^{4,5}$ In clinical practice, measurements are often restricted to the PACU, where currently pulse oximetry is the most important monitor of ventilation. However, oxygen saturation is not a direct measure of ventilation (but a measure of gas exchange in the lungs), and supplemental oxygen may obscure or delay the detection of respiratory events when oxygen saturation is used as a proxy for respiratory depression. ${ }^{6}$
Correspondence: Albert Dahan Department of Anesthesiology, Leiden University Medical Center, H5-P, Leiden 2300 RC, the Netherlands

Tel+3| 7| 526230 I

Email a.dahan@lumc.nl 
Given these facts, continuous measurement of ventilation, rather than oxygen saturation, is presumably a more accurate tool to detect respiratory events, and also to prevent such events. ${ }^{2,4,7,8}$ A practical method to determine breathing activity is to measure respiratory rate (RR). RR can be assessed by multiple methods, including intermittent manual counting of thoracic movement and electrocardiography (ECG)-derived technologies, which are both error-prone and may lead to the underdetection of respiratory events. ${ }^{9}$ For example, upper-airway obstruction is often not detected, due to the persistence of thoracic movements. Continuous monitoring of respiration is thus best accomplished by electronic devices detecting airflow at the mouth, as this gives a noninvasive indication of gas movement into and out of the lungs.

In the current study we measured RR in postoperative patients using the RespiR8 (Anaxsys, Send, UK), which was developed to measure RR based on the humidity of exhaled air. In a previous validation study, we showed that the RespiR8 monitor is as reliable as capnometry to measure $\mathrm{RR}$ in the range observed in clinical practice. ${ }^{9}$ Here, we measured RR during the 6 hours following surgery in the PACU, as well as on the ward. We tested an elderly population, 60 years of age and older, as there is evidence that elderly patients are at an elevated risk of respiratory events following opioid administration. ${ }^{10}$ Our main aim was to use continuous RR monitoring of the postoperative patient using the RespiR8 device in the PACU and on the ward to quantify the occurrence of respiratory events, split into bradypneic (RR 1-6 breaths/minute) and apneic (RR 0 breaths/minute for at least 1 minute) events. Additionally, we aimed to identify patient risk factors, such as neck circumference or body-mass index, that could predict the occurrence of an event.

\section{Materials and methods Study design and population}

The protocol was approved by the Ethics Committee of Leiden University Medical Center (the Netherlands) and by the National Research Ethics Service Committee, East Midlands (UK). All study procedures were conducted according to Good Clinical Practice guidelines and adhered to the tenets of the Declaration of Helsinki. This was a multicenter study performed at two sites: LUMC, Leiden, the Netherlands, and Nottingham University Hospital NHS Trust (Nottingham, UK). The study has been registered in the Netherlands Trial Register (NTR4778).

Of 115 recruited patients at the preoperative screening facility, 80 patients were enrolled in the study. All 80 study participants provided oral and written informed consent prior to study procedures and prior to their scheduled surgery. Inclusion criteria were age $\geq 60$ years, elective surgery under general anesthesia for at least 60 minutes, and ability to read and understand the patient-information and consent form. Exclusion criteria were head, neck, and facial surgery or other conditions prohibiting application of the RespiR8 mask postoperatively. Possible risk factors for apnea were recorded, such as sex, body-mass index, neck circumference, history of snoring, or diagnosis of obstructive sleep apnea. It was expected that at least 50 subjects would be required to observe a significant proportion of patients with at least one apnea event.

\section{Measurements}

All patients were extubated in the operating theater and transported to the PACU. In the PACU, patients were connected to standard clinical monitoring equipment, including three-lead ECG and pulse oximetry. All patients received conventional clinical care in the PACU facility, and were discharged to the ward based on local protocol.

RR was measured automatically and continuously using the RespiR8 monitor. The device performs a calculation using a three-breath averaging algorithm, and is expressed as breaths/minute. The RespiR8 sensor is integrated in a standard oxygen face mask that is placed upon arrival in the PACU, but does not functionally require a flow of exogenous oxygen. In cases where supplemental oxygen was required (ie, oxygen saturation $<94 \%$ ), 5-10 L/minute oxygen was administered. Supplemental oxygen was stopped before patients were discharged to the ward. RR measurements continued on the ward, and were temporarily discontinued in cases of eating/drinking, talking, or during other conditions where the face mask was removed. A research nurse remained with the patient during the complete 6-hour study period. Data were digitally recorded as average breaths/minute, and each data point represents a 1-minute average. A visual alarm was triggered when bradypnea (1-6 breaths/minute) or apnea ( 0 breaths/minute) was detected.

\section{Statistical analysis}

Statistical testing was performed using GraphPad Prism 6 (GraphPad Software, La Jolla, CA, US) and SPSS 20 (IBM, Armonk, NY, US). Data are presented as average \pm standard deviation or median (interquartile range), unless otherwise stated. Bradypnea was defined as an RR of $1-6$ breaths/minute, and apnea was defined as an absence of inspiratory flow at the mouth for at least 1 minute. Comparisons were made by 
Student's $t$-tests or nonparametric tests, and linear regression was used to correlate described variables. $P$-values $<0.05$ were considered significant.

\section{Results}

Of the 80 patients enrolled in the study, data from 68 patients were able to be used for analysis. One patient was admitted to the intensive care unit directly after surgery and thus excluded; data acquisition failed in four patients, and seven patients withdrew their informed consent postoperatively. Characteristics of the remaining 68 patients are given in Table 1. Ten patients were recruited at the UK site, and 58 patients were recruited at the Netherlands site.

Table I Patient characteristics, surgery, and pain relief

\begin{tabular}{ll}
\hline Number of patients analyzed & 68 \\
Sex, male/female & $38 / 30$ \\
Age (years) & $70[60-83]$ \\
Body-mass index $\left(\mathrm{kg} / \mathrm{m}^{2}\right)$ & $26.7[17.2-40 . \mathrm{I}]$ \\
Weight $(\mathrm{kg})$ & $79.6(\mathrm{I3.4})$ \\
Neck circumference (cm) & $38.7(4.4)$ \\
History of snoring, $\mathrm{n}(\%)$ & $38(56)$ \\
Diagnosis of obstructive sleep apnea, $\mathrm{n}(\%)$ & 0 \\
ASA status, $\mathrm{n}$ (\%) & \\
ASA I & $6(9)$ \\
ASA II & $57(84)$ \\
ASA III & $4(6)$ \\
Unknown & 1 (I) \\
Type of surgery, $\mathbf{n}$ (\%) & \\
General & $39(57)$ \\
Orthopedic & $11(16)$ \\
Urology & $15(22)$ \\
Gynecology & $3(4)$ \\
Anesthesia technique & \\
General & 68 \\
General + epidural/local & 19 \\
Type of anesthesia &
\end{tabular}

Note: Values expressed as mean (SD) or mean [range], unless otherwise stated. Abbreviations: ASA, American Society of Anesthesiologists; IV, intravenous; OT, operating theater; PACU, postanesthesia care unit; PCA, patient-controlled analgesia.
All patients received general anesthesia; 19 patients (28\%) received an additional epidural or local technique. These 19 patients did not receive additional opioids in the operating theater or in the PACU. Of the remaining 49 patients, 35 (71\%) received intravenous morphine as an opioid for postoperative pain relief. Typically, they received an opioid loading dose during surgery and received further increments of morphine titrated to their need in the PACU. Five patients subsequently received an intravenous patientcontrolled morphine device; four of these patients used the device on the ward. Consequently, of the entire study population, only four patients used additional opioids on the ward. Patients who did not receive any opioids postoperatively were treated with acetaminophen and nonsteroidal antiinflammatory agents at regular intervals (see also Table 1). Patients who received a muscle relaxant during surgery were adequately monitored and effectively reversed with either sugammadex or neostigmine according to standardized clinical protocol.

No serious adverse events occurred during the study. Nine adverse events were recorded, which were all judged to be not study-related. Adverse events included nausea and the occurrence of moderate pain, all occurring on the ward. All events were treated and resolved within the study period: pain was treated with a nonsteroidal anti-inflammatory agent, nausea with antiemetic medication.

RR monitoring using the RespiR8 device was successful during the first 6 postoperative hours. Almost 24,000 RR data points were collected ( 408 hours, 6 hours per patient); 482 data points (ie, 482 minutes or $2 \%$ of measurement time) were missing, due to face-mask removal because of eating/ drinking or other unspecified reasons. A histogram with the distribution of data points is shown in Figure 1. The median RR was 13 (10-15, range 0-39). In total, 877 measurements (3.6\%) were bradypneic. On 262 data points, apnea was detected (RR 0 breaths/minute for at least 1 minute, 1.1\%). Examples of RR values in the PACU and on the ward are shown in Figure 2. This shows one patient without any events in the PACU or on the ward (Figure 2A), one patient with multiple bradypneic events in the PACU and on the ward (Figure 2B), and finally one patient with apneic events in the PACU and on the ward (Figure 2C). None of the included patients in this study required verbal stimulation to breathe, mechanical support of ventilation, tracheal intubation, or the need for an opioid-reversal agent.

Eleven patients (16\%) experienced no respiratory events, and 53 patients $(78 \%)$ experienced at least one bradypneic event during the 6-hour study, with a median number of 


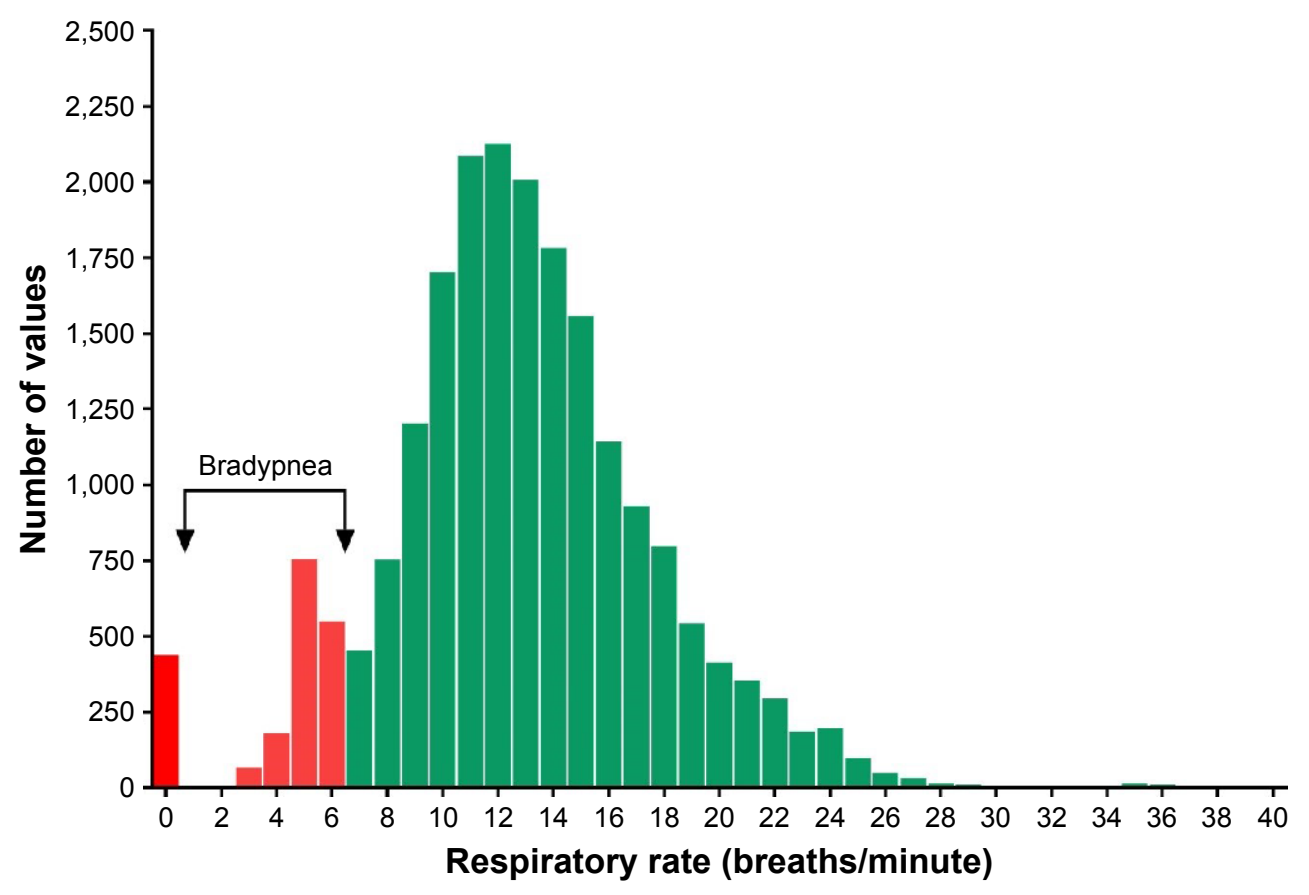

Figure I Observed respiratory rates.

Notes: Respiratory rate data were collected at I-minute intervals, and represent a data set of almost 24,000 observations from 68 patients.
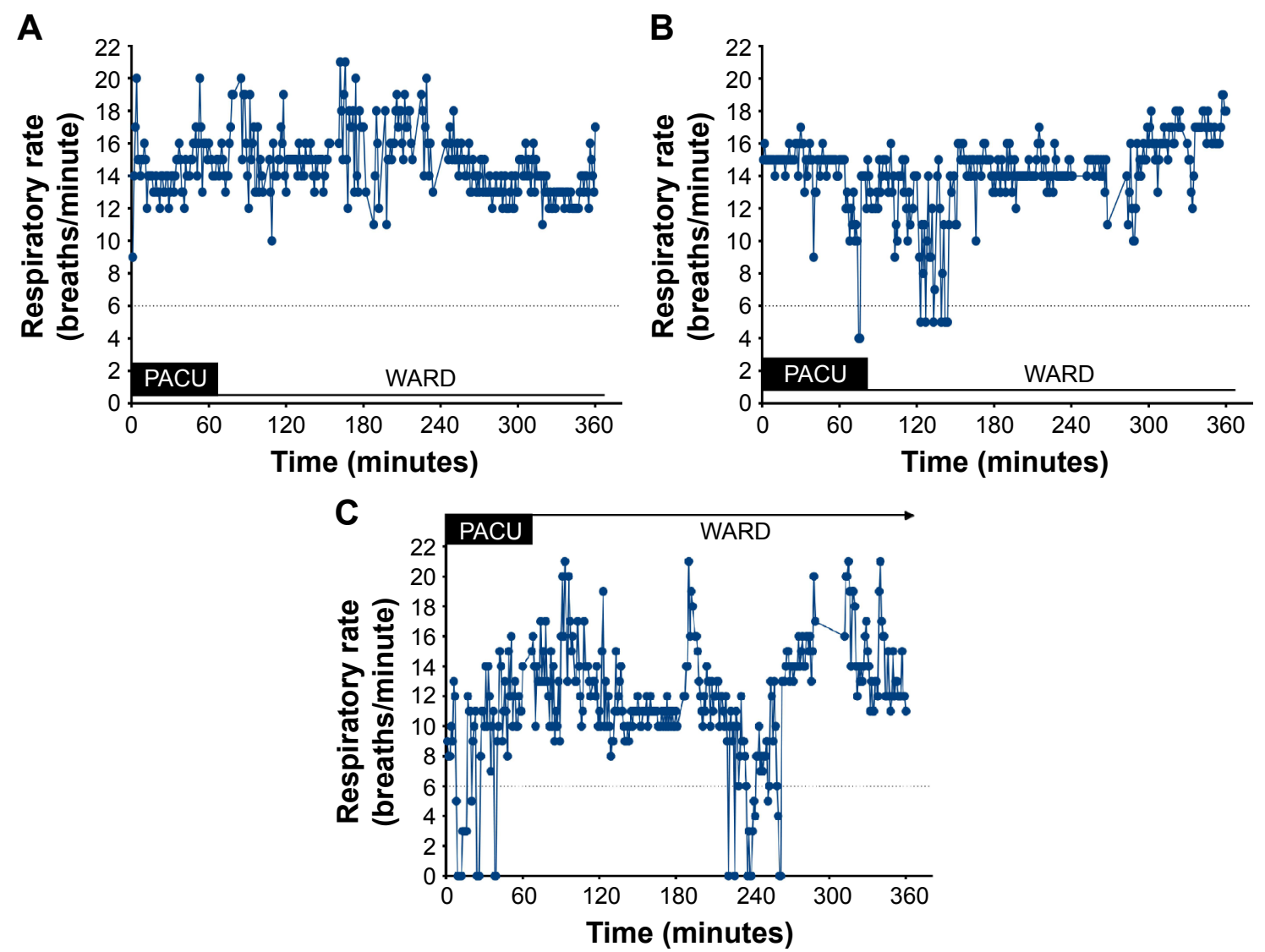

Figure 2 Examples of collected respiratory rate measurements in three patients $(\mathbf{A}-\mathbf{C})$.

Notes: Respiratory rate data were collected at I-minute intervals. Dotted, horizontal lines indicate the border for bradypnea. Time spent in the postanesthesia care unit (PACU) and on the ward is indicated. 
events per patient of ten (3.5-24). The median duration of these events was 1.4 minutes (1-1.6 minutes, range $1-4.3$ minutes). A total of 39 patients (57\%) experienced at least one apneic event, with a median number of events per patient of three (1-11, range 1-28). The median duration of an apneic event was 1.6 minutes (1-1.9 minutes, range $1-5$ minutes). A total of 35 patients (51\%) experienced both apneic and bradypneic episodes, while just four patients (6\%) had an apneic event without any bradypneic episodes. Finally, 18 patients $(26 \%)$ had only bradypneic events.

Patients who experienced bradypnea or apnea did not differ from patients without bradypnea or apnea with respect to age, body-mass index, sex, American Society of Anesthesiologists (ASA) status, positive history of snoring, type of surgery, or PACU time. Neck circumference was significantly larger in patients with apneic events than in patients without apneic events: $39.6(0.7) \mathrm{cm}$ versus $37.4(0.8) \mathrm{cm}(P<0.05)$, and neck circumference correlated significantly with the duration of apnea $(P<0.05)$. In contrast, neck circumference was not different for patients with or without bradypneic events: $38.7(0.6) \mathrm{cm}$ versus 38.8 (1) $\mathrm{cm}(P>0.05)$. None of the studied patient characteristics (age, body-mass index, sex, ASA status, positive history of snoring, type of surgery, PACU time) were correlated with the number or duration of bradypneic or apneic events (data not shown).

The occurrence of apneic and bradypneic events in the PACU was a predictor of events on the ward (bradypnea, $r^{2}=0.4, P<0.001$; apnea, $r^{2}=0.2, P<0.001$; Figure 3). Additionally, we observed a positive correlation between the occurrence of bradypneic events and apneic events $\left(r^{2}=0.4\right.$, $P<0.0001)$, indicative that both conditions frequently concurred. A weak correlation was observed between morphine dose and number of observed bradypneic $\left(r^{2}=0.2, P<0.05\right)$ and apneic $\left(r^{2}=0.3, P<0.05\right)$ events in the PACU, but not to the number of events on the ward (Figure 4). Finally, we observed no difference in the number of apneic and bradypneic events in patients who had received general anesthesia combined with an epidural or local block and patients who received just general anesthesia (Mann-Whitney test, $P>0.05)$.

\section{Discussion}

We studied RRs in postoperative patients aged 60 years and older following major elective surgery in the PACU and on the ward using the RespiR8 device. Continuous and reliable RR measurements were obtained during the first 6 postoperative hours for $98 \%$ of the time; data loss because of face-mask removal occurred in $2 \%$ of measurements. A respiratory event occurred in $84 \%$ of patients, with frequent bradypneic and apneic events in $78 \%$ and $57 \%$ of patients, respectively, with a median of 16.6 bradypneic events per patient and 6.6 apneic events per patient.

Various techniques to measure RR continuously in spontaneously breathing patients have been developed in recent years. These techniques include $\mathrm{CO}_{2}$ measurements at the mouth and nose, measurement of acoustic signals on the skin of the throat, impedance measurements from ECG, and measurements of thoracic and abdominal wall movement. ${ }^{1-16}$
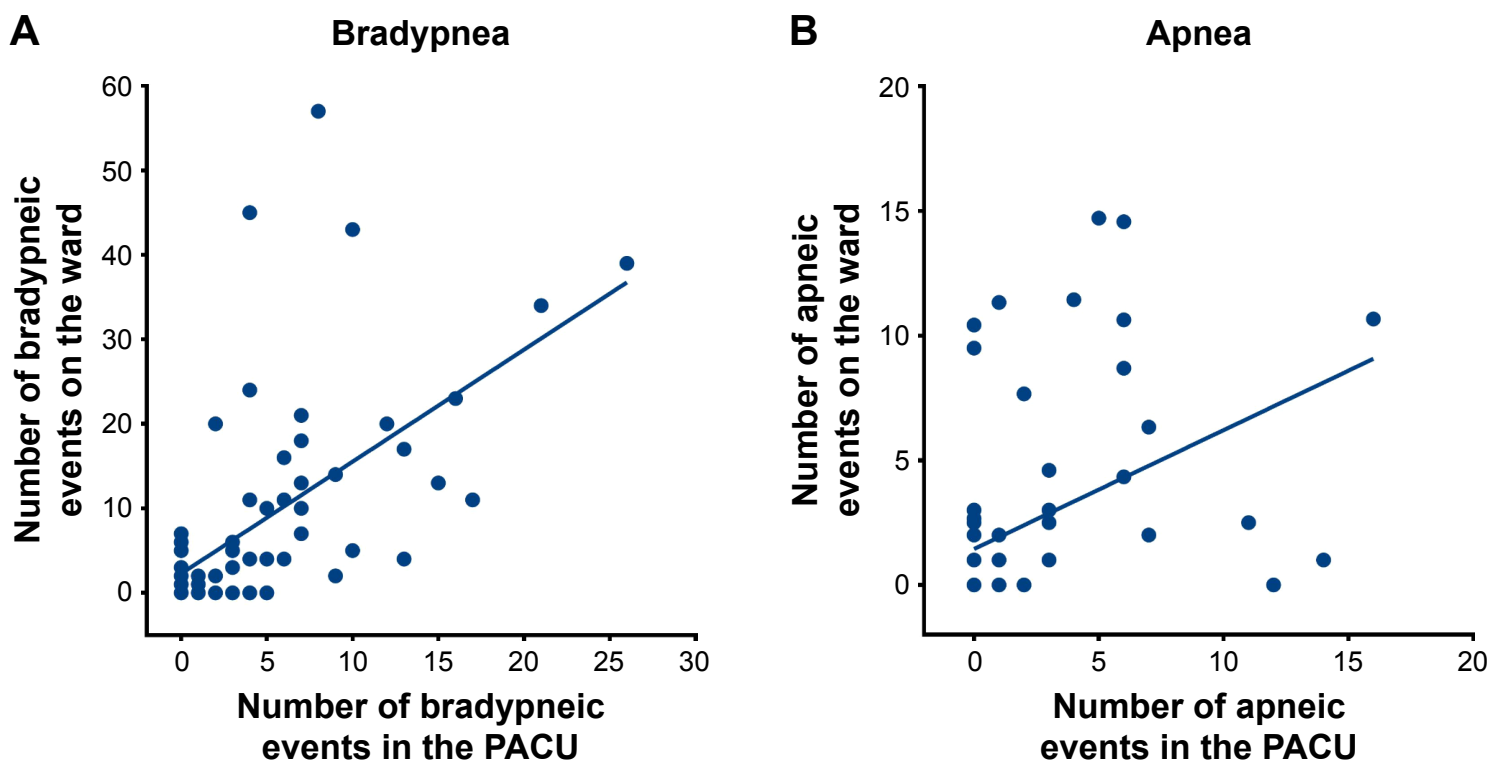

Figure 3 Correlation between respiratory events measured in the postanesthesia care unit (PACU) and on the ward. Notes: (A) Bradypneic events, $r^{2}=0.4(P<0.000 \mathrm{I})$; (B) apneic events, $r^{2}=0.2(P<0.00 \mathrm{I})$. 

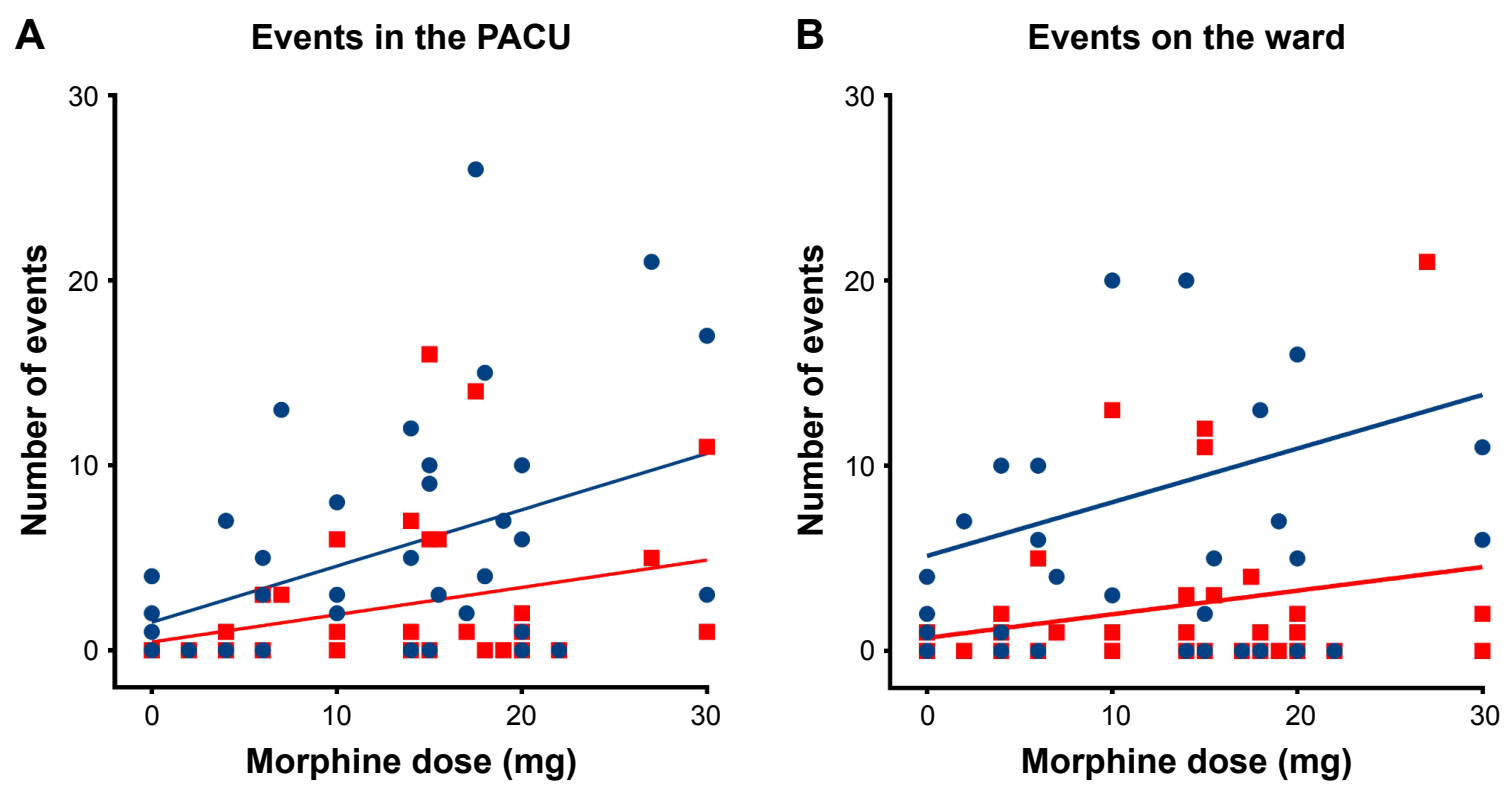

Figure 4 Correlation between morphine dose administered in the operating theatre and in the post-anesthesia care unit (PACU).

Notes: Number of bradypneic (blue circles) and apneic (red squares) events in the PACU $(\mathbf{A})$ and on the ward $(\mathbf{B})$. (A) Bradypnea, $r^{2}=0.2(P<0.05)$; apnea, $r^{2}=0.3(P<0.05)$. (B) Bradypnea, $r^{2}=0.1$ ( $\left.P>0.05\right)$; apnea, $r^{2}=0.2(P>0.05)$.

Recently a new noninvasive technique was published that uses a radar system mounted above the patient's bed to measure RR. ${ }^{17}$ In this study, we used the RespiR8, a small and mobile device that relies on the detection of humidity in the exhaled air (see Niesters et $\mathrm{al}^{9}$ for a detailed description of the RespiR8 measurement technique). The current study, as well as our previous validation study, shows that the device allows long-term reliable assessment of RR. ${ }^{9}$ In our sample of 68 patients, the device detected multiple events of low RR and events with complete cessation of airflow at the mouth. It is important to realize that the system does not provide information on the cause or location (central or obstructive) of the respiratory event.

Additionally, it is important that although respiratory events were frequent, they were short-lived and did not necessitate any intervention, such as verbal stimulation to breathe, mechanical ventilation, intubation, admission to the intensive care unit (ICU), or any pharmacological intervention. We relate this to the fact that our sample was relatively small and opioid use on the ward minimal. Our data are supportive of the recommendation from national anesthesia societies for the continuous monitoring of RR in spontaneously breathing patients who receive potent opioids for pain relief., ${ }^{7,8}$ An important observation was that early respiratory events in the PACU predict events on the ward. Since opioid use on the ward was limited in our patient population, this observation suggests that in some patients, respiratory instability persists beyond their stay in the PACU. Such prolonged periods of instability may be related to underlying disease, surgical stress, residual anesthetics and relaxants, opioid use in the PACU, or any combination of these factors. Irrespective of the cause, the early detection of respiratory events can serve as a clinical warning sign, and especially the population with early respiratory instability may require RR monitoring on the ward, and also in patients who do not consume large amounts of opioids.

The amount of morphine use was only weakly correlated with the occurrence of respiratory events in the PACU; no correlation was observed for events on the ward. The latter was expected, given the fact that opioid use on the ward was restricted to just four patients. The weak correlation is somewhat surprising, given the fact that the average morphine dose was relatively high $(0.22 \mathrm{mg} / \mathrm{kg})$. Still, as suggested, factors other than opioid consumption alone may have played a causal role in the generation of respiratory events in our patient population. Whether our findings are related to the age-group we tested remains unknown. We recently observed more frequent apneic events in an elderly population following oxycodone $20 \mathrm{mg}$ intake (with and without additional ethanol) relative to a younger population. ${ }^{10} \mathrm{We}$ relate these latter findings as well as our current results to higher opioid sensitivity and altered pharmacokinetics in the elderly, but additionally to alterations in the metabolic state, greater stress response to surgery and anesthesia, loss of resilience, reduced circulating volume and lean-muscle mass, and reduced reserve capacity in the elderly. ${ }^{18-20}$ These 
factors may be more pronounced in a certain subcategory of elderly patients ("the frail"), which would account for the occurrence of respiratory events in many but not all patients. At present, we have no reliable way of predicting which elderly patients are at increased risk of postoperative respiratory events. These findings warrant intensified, continuous monitoring of the respiratory status of postoperative elderly patients. Further studies are needed to understand fully the age effect on the control of breathing following major surgery under general anesthesia.

Patients who experienced apneic events had larger neck circumference than patients who did not have such events. In contrast, no such dichotomy was observed for bradypneic events. Neck circumference is an important predictor of upper-airway obstruction in sleep and sedation. ${ }^{21}$ This explains why patients with relatively greater neck circumference had more apneic episodes, most probably related to upper-airway obstruction, but not bradypneic events, as these are a measure of central respiratory instability.

Our study has a number of limitations. We aimed to evaluate the clinical use of the RespiR8 device and quantify the occurrence of respiratory events. Consequently, this study was not powered to detect any clinical consequences of the recorded respiratory events. Oxygen saturation and the use of supplemental oxygen in the PACU or on the ward were not recorded. Additionally, there was no assessment of mental state to detect the occurrence of delirium or postoperative cognitive decline. It is thus unclear what the clinical significance of the recorded events was. Future, longitudinal studies of respiratory events in elderly postoperative patients should address these issues. Also, the use of opioids on the ward was particularly low. This may have accounted for the absence of severe pain on the ward, but we cannot exclude that the patient's age played some role as well (eg, fear of opioid use on the ward in elderly patients). It seems likely that we would have found a stronger correlation between opioid use and respiratory events with a higher total dose of administered morphine. This has been observed in patients aged 65 years and older on intravenous patient-controlled morphine. Interestingly, in that study, respiratory events ( $\mathrm{RR}<10$ breaths/minute for at least 2 minutes) occurred in $78 \%$ of patients. ${ }^{4}$ This incidence is remarkably similar to data obtained in our study, and suggests that there are inherent opioid-dependent and nonopioid-dependent factors that predispose to postoperative respiratory events. Additionally, the RespiR8 sensor is based on exhaled humidity. ${ }^{9}$ The presence of shallow (opioid-induced) breathing patterns or the application of (dry) oxygen flow may change RespiR8 performance, and should be taken into account when interpreting our data. Finally, periods of sleep or sedation were not recorded. Both sleep and sedation can affect breathing patterns, and could be informative when analyzing longitudinal RRs.

In conclusion, this is the first long-term data-collection study on postoperative RRs in adults aged 60 years and older using the RespiR8 system. Almost $80 \%$ of patients experienced at least one bradypneic period during the 6-hour postoperative period, and almost $60 \%$ of patients had at least one apnea event. These events occurred well into the postoperative period, even in the absence of opioid administration. Known risk factors did not predict which patients were at risk for postoperative respiratory depression. Our data demonstrate the magnitude of respiratory disturbances in a postoperative elderly population, which may relate to presently unknown patient specific factors. Continuous respiratory monitoring during this time frame is warranted in this vulnerable patient population.

\section{Acknowledgment}

This work was supported in part by Anaxsys Technology Ltd, Send, UK.

\section{Author contributions}

All authors contributed toward protocol writing, data acquisition, data analysis, drafting and critically revising the paper, gave final approval of the version to be published, and agree to be accountable for all aspects of the work.

\section{Disclosure}

The authors report no conflicts of interest in this work. Albert Dahan is chaiman of the Leiden University Medical Center Institutional Review Board. He was not involved in the ethical review of this study.

\section{References}

1. Dahan A, Aarts L, Smith TW. Incidence, reversal, and prevention of opioid-induced respiratory depression. Anesthesiology. 2010;112(1): 226-238.

2. Niesters M, Overdyk F, Smith T, Aarts L, Dahan A. Opioid-induced respiratory depression in paediatrics: a review of case reports. Br J Anaesth. 2013;110(2):175-182.

3. Overdyk F, Dahan A, Roozekrans M, van der Schrier R, Aarts L, Niesters M. Opioid-induced respiratory depression in the acute care setting: a compendium of case reports. Pain Manag. 2014;4(4):317-325.

4. Overdyk FJ, Carter R, Maddox RR, Callura J, Herrin AE, Henriquez C. Continuous oximetry/capnometry monitoring reveals frequent desaturation and bradypnea during patient-controlled analgesia. Anesth Analg 2007;105(2):412-418.

5. Sun Z, Sessler DI, Dalton JE, et al. Postoperative hypoxemia is common and persistent: a prospective blinded observational study. Anesth Analg. 2015 121(3):709-715. 
6. Niesters M, Mahajan RP, Aarts L, Dahan A. High-inspired oxygen concentration further impairs opioid-induced respiratory depression. BrJAnaesth. 2013;110(5):837-841.

7. Stoelting RK, Overdyk F. Essential monitoring strategies to detect clinically significant drug-induced respiratory depression in the postoperative period: conclusions and recommendations. 2011. Available from: http://www.apsf.org/newsletters/html/2011/fall/01_opioid.htm. Accessed June 29, 2017.

8. Weinger MB, Lee L. No patient shall be harmed by opioid-induced respiratory depression. APSF Newsl. 2011;26(2):21-28.

9. Niesters M, Mahajan R, Olofsen E, et al. Validation of a novel respiratory rate monitor based on exhaled humidity. Br J Anaesth. 2012;109(6): 981-989.

10. van der Schrier R, Roozekrans M, Olofsen E, et al. Influence of ethanol on oxycodone-induced respiratory depression: a dose-escalating study in young and elderly individuals. Anesthesiology. 2017;126(3): 534-542.

11. Anderson W, Brock-Utne AJ, Brock-Utne JG, Brodsky JB. Evaluation of a respiratory rate monitor in postsurgical patients. J Clin Anesth. 1992; 4(4):289-291.

12. Drummond GB, Bates A, Mann J, Arvind DK. Validation of a new non-invasive automatic monitor of respiratory rate for postoperative subjects. Br J Anaesth. 2011;107(3):462-469.

13. Frasca D, Geraud L, Charriere JM, Debaene B, Mimoz O. Comparison of acoustic and impedance methods with mask capnometry to assess respiration rate in obese patients recovering from general anaesthesia. Anaesthesia. 2015;70(1):26-31.
14. Gaucher A, Frasca D, Mimoz O, Debaene B. Accuracy of respiratory rate monitoring by capnometry using the Capnomask in extubated patients receiving supplemental oxygen after surgery. Br J Anaesth. 2012;108(2): 316-320.

15. Mimoz O, Benard T, Gaucher A, Frasca D, Debaene B. Accuracy of respiratory rate monitoring using a non-invasive acoustic method after general anaesthesia. Br J Anaesth. 2012;108(5):872-875.

16. Nilsson L, Johansson A, Kalman S. Monitoring of respiratory rate in postoperative care using a new photoplethysmographic technique. J Clin Monit Comput. 2000;16(4):309-315.

17. van Loon $\mathrm{K}$, Breteler MJ, van Wolfwinkel L, et al. Wireless noninvasive continuous respiratory monitoring with FMCW radar: a clinical validation study. J Clin Monit Comput. 2016;30(6):797-805.

18. Aubrun F. Management of postoperative analgesia in elderly patients. Reg Anesth Pain Med. 2005;30(4):363-379.

19. Rivera R, Antognini JF. Perioperative drug therapy in elderly patients. Anesthesiology. 2009;110(5):1176-1181.

20. Dodds C, Kumar CM, Veering BT. Oxford Textbook of Anesthesia for the Elderly Patient. Oxford: Oxford University Press; 2014.

21. Cizza G, de Jonge L, Piaggi P, et al. Neck circumference is a predictor of metabolic syndrome and obstructive sleep apnea in short-sleeping obese men and women. Metab Syndr Relat Disord. 2014;12(4):231-241.
Therapeutics and Clinical Risk Management

\section{Publish your work in this journal}

Therapeutics and Clinical Risk Management is an international, peerreviewed journal of clinical therapeutics and risk management, focusing on concise rapid reporting of clinical studies in all therapeutic areas, outcomes, safety, and programs for the effective, safe, and sustained use of medicines. This journal is indexed on PubMed Central, CAS,

\section{Dovepress}

EMBase, Scopus and the Elsevier Bibliographic databases. The manuscript management system is completely online and includes a very quick and fair peer-review system, which is all easy to use. Visit http://www.dovepress.com/testimonials.php to read real quotes from published authors. 